\title{
Physician-controlled costs: the choice of equipment used for atrial fibrillation ablation
}

\author{
Roger A. Winkle • R. Hardwin Mead • Gregory Engel • \\ Melissa H. Kong • Rob A. Patrawala
}

Received: 5 September 2012 /Accepted: 13 January 2013 /Published online: 14 March 2013

(C) The Author(s) 2013. This article is published with open access at Springerlink.com

\begin{abstract}
Purpose Atrial fibrillation (AF) ablation uses expensive technology and equipment. Physicians have considerable latitude over equipment choice. Average Medicare reimbursement is $\$ 10,338$ for uncomplicated $\mathrm{AF}$ ablations. The purpose of this study is to evaluate the cost of special equipment chosen by physicians to perform $\mathrm{AF}$ ablation. Methods We obtained the list price cost of special capital equipment and of disposable equipment (intracardiac ultrasound probes, transseptal needles/sheaths, and ablation/recording catheters) commonly used for radiofrequency (RF) $\mathrm{AF}$ ablation. We also evaluated the equipment cost of using robotic magnetic navigation and cryoablation. Then we evaluated costs for several physician equipment choice scenarios.

Results Using open irrigated-tip catheters, the lowest estimated cost-per-case for manual RF ablation of AF was $\$ 6,637$, and the highest estimated cost of manual RF ablation was $\$ 12,603$. Assuming $200 \mathrm{AF}$ ablations/year and a 6year magnet life, the cost-per-case of using magnetic navigation ablation ranged from $\$ 12,261-\$ 15,464$. The costper-case using cryoballoons alone ranged from $\$ 12,847$ $\$ 15,320$, and if focal cryoablation or RF touch-up is needed, cryoablation cost increased to $\$ 15,942-\$ 22,284$.

Conclusions Physicians have many choices in AF ablation equipment. Equipment costs in our arbitrary scenarios range from $\$ 6,637$ to $\$ 22,284$ per case. More important than the specific cost of each scenario is the concept that these are
\end{abstract}

R. A. Winkle $(\bowtie) \cdot$ R. H. Mead · G. Engel • M. H. Kong •

R. A. Patrawala

From Silicon Valley Cardiology and Sequoia Hospital,

1950 University Avenue, Suite 160, E. Palo Alto, CA 94303,

Redwood City, USA

e-mail: rawinkle@aol.com physician-driven costs, and as such, physicians will need to determine if more expensive technologies increase procedural efficacy and/or patient safety enough to justify the greater procedural equipment costs.

Keywords AF ablation · Ablation costs · Atrial fibrillation

\section{Introduction}

Atrial fibrillation (AF) ablation is an increasingly widely used treatment for patients with symptomatic AF refractory to antiarrhythmic drug therapy $[1,2]$. Studies reporting on AF ablation techniques [3-5], outcomes [6-11], complications [12], and a variety of new technologies for performing $\mathrm{AF}$ ablations are continuously published. These procedures can be lengthy, involve numerous physician and nonphysician personnel, and are performed in expensive electrophysiology laboratories, employing a wide variety of specialized technologies. Little information is currently available on the cost of the equipment utilized to perform $\mathrm{AF}$ ablations. These procedures require mapping equipment, mapping catheters, robotic $[13,14]$ and/or manual catheter manipulation, and a variety of ablation catheters utilizing radiofrequency (RF), laser, and ultrasound energy sources for tissue heating and cryoablation for tissue cooling. Our study examines the cost of special equipment and catheters used to perform AF ablations with particular emphasis on the array of equipment choices available to physicians and their impact on AF ablation costs. Although we examine the costs of several specific ablation equipment scenarios, the details of each scenario is not as important as the concept that physicians have a wide range of choices for performing AF ablation with a wide range of costs. 


\section{Methods}

We obtained list prices in US\$ from manufacturers of equipment commonly utilized to perform AF ablations in the US. We assumed a standard electrophysiology laboratory was in place including fluoroscopy, external defibrillators, intravenous infusion pumps, oxygen saturation monitors, equipment to measure activated clotting times, echocardiography machines with transesophageal echocardiography probes, and equipment for multichannel recording and stimulation. We did not include the facility and overhead costs, the cost of post-ablation monitoring, personnel costs, or physician reimbursment, as these would be expected to be relatively independent of the equipment utilized to perform the AF ablation. Cost analysis was performed by Dr. Winkle.

\subsection{Capital equipment}

We examined the costs of a three-dimensional (3D) electroanatomical mapping system: Biosense Webster's (Diamond Barr, CA, USA) Carto ${ }^{\text {TM }} 3$ and St. Jude Medical's (St. Paul, MN, USA) EnSite Velocity ${ }^{\mathrm{TM}}$ system. We estimated the cost of special capital equipment required for intracardiac echocardiography (ICE) to facilitate transseptal puncture and the cost of RF generators and irrigation pumps for RF ablation catheters and RF transseptal needles. Maintenance contract costs were included assuming a 5-year life for nonnavigation capital equipment.

\subsection{Disposable catheters and cables}

We examined the list price for open and closed irrigated-tip RF ablation catheters including those with unidirectional or bidirectional steering mechanisms, those with technology required for the Biosense Webster Carto ${ }^{\text {TM }} 3$ mapping system and for the Stereotaxis (St. Louis, MO, USA) NIROBE $^{\circledR}$ Remote Magnetic Navigation System (MNS). We obtained list prices for coronary sinus (CS) duodecapolar catheters, circular mapping catheters for confirming pulmonary vein (PV) isolation with both fixed- and variablediameter circles, and for standard transseptal needles and the Baylis (Montreal, QC, Canada) NRGTM RF needle. We included the cost of cables for the ablating catheters and disposable patches for the 3D mapping systems.

\subsection{Costs comparison of open irrigated-tip RF ablation} catheters

We compared cost differences for the lowest- and highestcost open irrigated-tip RF ablation catheters and the associated cables and patches for the two dominant brands, Biosense Webster and St. Jude Medical.

\subsection{Magnetic navigation equipment}

The cost of an installed Stereotaxis $\mathrm{NIROBE}^{\circledR}$ Remote MNS including maintenance contracts was determined assuming a 6-year magnet life. This cost did not include structural room changes required for installation. We included the cost of disposable driving mechanisms for the MNS.

\subsection{Cryoablation}

The cost of the Medtronic (Minneapolis, MN, USA) CryoConsole unit including maintenance contracts was determined using a 5-year lifespan. We included the cost of the disposable Arctic Front ${ }^{\circledR}$ cryoablation balloon catheters, cables, FlexCath ${ }^{\circledR}$ sheaths, the Achieve ${ }^{\mathrm{TM}}$ circular mapping catheter, and the Freezor ${ }^{\circledR}$ MAX linear cryoablation catheter for touch-up ablation.

\subsection{Ablation scenarios}

We evaluated several common clinical scenarios for physician equipment choices for AF ablation:

1. Open irrigated-tip RF ablation;

2. Magnetic robotic navigation RF ablation;

3. Balloon cryoablation without touch-up;

4. Balloon cryoablation with linear cryoablation or open irrigated-tip RF touch-up.

For each scenario, the lowest and highest costs of equipment choices were estimated. For all scenarios, we assumed the use of ICE for transseptal puncture, a duodecapolar CS catheter, a circular mapping catheter to verify PV isolation, and a 3D mapping system (except cryoballoon ablation without touch-up). For scenario 1, open irrigated-tip RF ablation, we did not include the small capital cost of the mapping system as these are available in most EP labs and are used frequently for other non-AF ablations. We assumed no resterilization of mapping or ablation catheters, ten reuses of passive recording cables for mapping catheters, and no reuse of active RF carrying cables. Cable reprocessing costs were not included nor were costs of RF generators or irrigation pumps as these were relatively equal across ablation systems and represented a small incremental cost/case.

\section{Results}

3.1 Medicare reimbursement for AF ablation

Uncomplicated AF ablations are billed under DRG 251 with an average reimbursement of $\$ 10,338$ [15]. 
3.2 Costs of capital equipment, reusables, and disposables

Table 1 shows the cost of special capital equipment for AF ablation. The 5-year cost for the mapping systems/maintenance contracts is $\$ 375,000$ for the Biosense Webster Carto $^{\text {TM }} 3$ and \$495,000 for the St. Jude EnSite Velocity ${ }^{\text {TM }}$. Phased array ICE catheters use standard ultrasound machines; however, the rotational ultrasound catheter (Ultra ICE ${ }^{\mathrm{TM}}$, Boston Scientific, Natick, MA, USA) requires a special machine $\left(\mathrm{iLab}^{\circledR}\right)$ with a 5-year cost of $\$ 131,400$. The 5-year cost ranged from \$33,000-\$67,000 for RF generators and $\$ 12,000-\$ 19,000$ for irrigation pumps.

The cost of ablation and mapping catheters and their respective cables and patches are listed in Table 2 along with the costs of ICE catheters and transseptal needles and sheaths. The cost of unidirectional open irrigated-tip catheters ranged from $\$ 1,500-\$ 2,800$. Bidirectional catheters were more expensive ranging from $\$ 1,700-\$ 3,000$. Catheters compatible with the St. Jude Ensite Velocity ${ }^{\mathrm{TM}}$ system ranged from $\$ 1,500-\$ 1,900$, and the special navigation catheters required by Carto $^{\text {TM }} 3$ cost $\$ 2,800$ $\$ 3,000$. Ablation cables ranged from $\$ 170-\$ 400$. The navigation patches from Biosense Webster were $\$ 420$, and those from St. Jude were $\$ 1,045$. The Medtronic Arctic Front ${ }^{\circledR}$ cryoballoon cost $\$ 6,500$. Its cable and umbilical cable cost $\$ 215$ and $\$ 145$. The Freezor ${ }^{\circledR}$ MAX focal cryoablation catheter cost $\$ 3,095$. The MNS requires special navigation catheters and a disposable catheter advancement system costing $\$ 1,200$. Unidirectional CS catheters cost from $\$ 1,150-\$ 1,200$ and the Boston Scientific (Blazer ${ }^{\circledR}$ DX-20) bidirectional duodecapolar catheter was slightly more expensive at $\$ 1,400$.

Circular mapping catheters had more variation in cost. Fixed-diameter circular decapolar catheters ranged from $\$ 1,200-\$ 1,540$. Fixed-diameter circular duodecapolar catheters ranged from $\$ 1,400-\$ 1,700$ with all cables costing $\$ 250$. Variable diameter circular decapolar and duodecapolar catheters ranged from $\$ 1,700-\$ 1,800$, and the cables cost \$110-\$250. The MNS requires a circular catheter advancement system costing \$250. The cryoballoon utilizes a "through-the-balloon" circular mapping catheter (Achieve ${ }^{\mathrm{TM}}$ ) costing $\$ 1,500$ which can only be used with the cryoballoon and requires a $\$ 250$ cable.

The lowest cost ICE is the Boston Scientific Ultra ICE $^{\mathrm{TM}}$ rotational catheter costing $\$ 1,050$. The most expensive are the phased-array catheters costing $\$ 2,640$ $\$ 2,800$. Although the rotational ICE catheter costs $\$ 1,590$ less than the phased array, it requires a separate ultrasound processor $\left(\mathrm{iLab}^{\circledR}\right)$ at a 5 -year cost of $\$ 131,400$; therefore, it takes 82.6 cases to begin saving on each rotational ICE catheter.

Transseptal needle costs range from $\$ 175$ for standard needles to $\$ 495$ for the Baylis $\mathrm{NRG}^{\mathrm{TM}} \mathrm{RF}$ needle which requires a $\$ 150$ cable. Non-steerable transseptal sheaths from range from \$175-\$225. The steerable St. Jude Agilis $^{\mathrm{TM}}$ sheath costs $\$ 999$. Cryoablation catheters require special FlexCath ${ }^{\circledR}$ steerable sheaths costing \$1,200-\$1,800.

The total cost for short sheaths, IV tubing, air filters, needles, syringes, defibrillation pads, and IV infusion materials is $\$ 434.90$ and were not included in the following ablation scenarios as they are roughly the same for all.

\subsection{Cost comparison of open irrigated-tip RF ablation catheters}

Figure 1 shows the highest- and lowest-cost RF ablation catheters from St. Jude and Biosense Webster. The lowestcost St. Jude RF ablation catheter including cables and navigation patches is the Cool $\mathrm{Path}^{\mathrm{TM}}$ unidirectional catheter costing \$2,840. The lowest-cost Biosense Webster navigation RF ablation catheter with cables and navigation patches is the ThermoCool ${ }^{\circledR}$ unidirectional catheter which costs $\$ 870$ more at $\$ 3,710$. Because the St. Jude EnSite Velocity ${ }^{\mathrm{TM}}$ mapping system costs $\$ 84,500$ more to purchase than the Carto ${ }^{\mathrm{TM}} 3$, it requires 97.1 cases to begin saving with the

Table 1 Cost of capital equipment including initial cost and 5-year maintenance contract costs in US\$

\begin{tabular}{|c|c|c|c|c|c|c|c|c|c|c|}
\hline \multirow{2}{*}{$\begin{array}{l}\begin{array}{l}\text { Capital } \\
\text { equipment }\end{array} \\
\text { Mapping system }\end{array}$} & \multicolumn{2}{|c|}{ Biosense Webster } & \multicolumn{2}{|l|}{ St. Jude } & \multicolumn{2}{|c|}{ Boston Scientific } & \multicolumn{2}{|l|}{ Baylis } & \multicolumn{2}{|l|}{ Medtronic } \\
\hline & Carto $^{\mathrm{TM}} 3$ & $\$ 375,000^{\mathrm{a}}$ & $\begin{array}{l}\text { EnSite } \\
\text { Velocity }\end{array}$ & $\$ 459,900^{\mathrm{a}}$ & & & & & & \\
\hline Ultrasound & & & & & $\mathrm{iLab}{ }^{\circledR}$ & $\$ 131,400^{\mathrm{b}}$ & & & & \\
\hline RF generator & Stockert & $\$ 48,300$ & IBI 1500 T9 & $\$ 33,000$ & Maestro ${ }^{\circledR}$ & $\$ 67,000$ & RFP-100 & $\$ 26,384$ & & \\
\hline Irrigation pump & CoolFlow ${ }^{\circledR}$ & $\$ 12,000$ & $\begin{array}{l}\text { Cool } \\
\text { Point }\end{array}$ & $\$ 19,000$ & CircuCool ${ }^{\circledR}$ & $\$ 12,750$ & & & & \\
\hline $\begin{array}{c}\text { Cryoablation } \\
\text { generator }\end{array}$ & & & & & & & & & Cryo-console & $\$ 265,000$ \\
\hline
\end{tabular}

${ }^{\mathrm{a}} \$ 84.500$ difference in mapping systems over 5 years

${ }^{\mathrm{b}}$ Required in addition to regular ultrasound to use Boston Scientific rotational intravascular ultrasound 
Table 2 Cost of catheters including required cables, patches, and robotic drive units in US\$

Ablation catheters, cables, patches, and equipment

Biosense Webster

Non-navigation Navigation Magnetic navigation

Closed irrigated tip

Closed irrigated tip cable

Closed irrigated tip tubing

Open irrigated tip unidirectional

Open irrigated tip bidirectional

Open irrigated stereotaxis

Catheter advancement system

Cable

Navigation patch

Ablation patch

Irrigation tubing

Cryoablation balloon

Cryoablation focal ablation

Cryoablation cable

Cryoablation coaxial umbilical

Mapping catheters, cables, patches, and equipment

Biosense Webster

Non-navigation

Duodecapolar unidirectional

$\$ 1,200$

Duodecapolar unidirectional cable

$\$ 250$

Duodecapolar bidirectional

Duodecapolar bidirectional cable

Circular fixed 10 pole

Circular fixed 20 pole

$\$ 1,200$

$\$ 1,400$

$\$ 250$

$\$ 1,800$

$\$ 1,800$

$\$ 250$

Circular variable 20 pole

Circular variable cable

Catheter advancement system

Intracardic echocardiography catheters, transseptal needles and cables, and transseptal sheaths

$\begin{array}{ll}\text { ICE catheter } & \begin{array}{l}\text { Biosense Webster } \\ \text { Transseptal needle }\end{array} \\ \begin{array}{l}\text { Transseptal RF cable } \\ \text { Transseptal sheath }\end{array} & \$ 175 \\ \begin{array}{l}\text { Transseptal sheath steerable } \\ \text { Cryoablation balloon sheath }\end{array} & \$ 175 \\ \text { Cryoablation focal sheath } & \end{array}$

$\$ 2,400^{\mathrm{b}}$

St. Jude Boston Scientific Stereotaxis Medtronic

$\$ 1,690$

$\$ 375$

$\$ 80$

$\$ 1,525$

$\$ 1,900$

$\$ 6,500^{\mathrm{c}}$

$\$ 3,095^{\mathrm{c}}$

$\$ 215^{\mathrm{c}}$

$\$ 145^{\mathrm{c}}$

St. Jude Boston Scientific Stereotaxis Medtronic

$\$ 1,500^{\mathrm{c}}$

$\$ 250^{\mathrm{c}}$
Navigation Magnetic navigation $\$ 1,200^{\text {a }} \$ 1,200^{\mathrm{b}}$

$\$ 250 \quad \$ 250$

$\$ 400 \quad \$ 400 \quad \$ 170$

$\$ 420 \quad \$ 420 \quad \$ 1,045$

$\$ 90 \quad \$ 90 \quad \$ 100$

$\$ 18 \quad \$ 12$

$\$ 1,150$

$\$ 220$

$\$ 1,400$

$\$ 250$

$\$ 1,540^{\mathrm{a}}$

$\$ 1,700^{\mathrm{a}}$

$\$ 250$

$\$ 1,700$

$\$ 110$

$\$ 1,200^{b}$

$\$ 1,700^{a}$

$\$ 1,800^{\text {a }}$

$\$ 250$
$\$ 250^{\mathrm{b}}$

St. Jude Boston Scientific Baylis Medtronic $\$ 2,640 \$ 1,050^{\mathrm{d}}$

$\$ 240 \quad \$ 495$

$\$ 150$
$\$ 225$

$\$ 999$

${ }^{\mathrm{a}}$ Required for Carto ${ }^{\mathrm{TM}} 3$ Mapping System

${ }^{\mathrm{b}}$ Required for magnetic navigation

${ }^{\mathrm{c}}$ Required for cryoablation

${ }^{\mathrm{d}}$ Requires separate echo machine for $\$ 131,400$ thus number needed to begin saving $=82.6$ cases

Cool Path ${ }^{\mathrm{TM}}$ compared with the ThermoCool ${ }^{\circledR}$. The most expensive St. Jude ablation catheter is the Saffire BLUTM bidirectional catheter, which costs $\$ 3,215$ with cables and navigation patches and is \$695 less than Biosense Webster's ThermoCool ${ }^{\circledR}$ bidirectional catheter with associated cables and navigation patches. Due to the higher cost of the St. 
Fig. 1 Highest and lowest estimated cost of RF open irrigated-tip ablation catheters from St. Jude and Biosense Webster

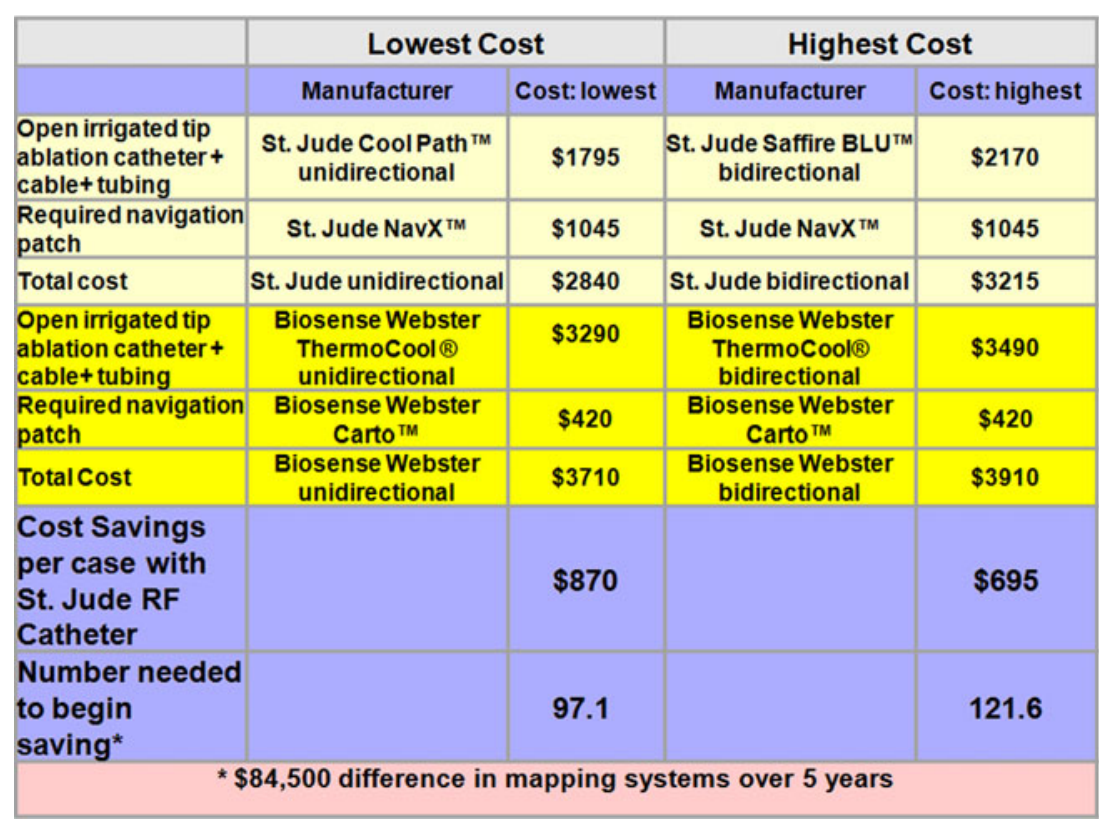

Jude EnSite Velocity ${ }^{\mathrm{TM}}$ mapping system, it requires 121.6 cases to begin saving the $\$ 695$ with the Saffire BLUTM ablation catheter.

\subsection{Estimated equipment costs for RF ablation}

Figure 2 shows the lowest and highest hypothetical cost scenarios for open irrigated-tip RF ablation. The lowestcost scenario is $\$ 6,637$, and the highest is $\$ 12,603$ which is
$89.9 \%$ more expensive than the lowest-cost RF equipment.

\subsection{Cost of magnetic navigation equipment}

The manufacturer's list price for the Stereotaxis NIROBE ${ }^{\circledR}$ Robotic MNS is $\$ 2,875,000$ with an annual maintenance contract of $\$ 104,000$ per year for a total 6-year cost of $\$ 3,395,000$. Assuming 200 cases/year, the robotic MNS costs $\$ 2,829$ per case. It requires a disposable $\$ 1,200 /$ case
Fig. 2 Lowest and highest estimated cost of equipment for performing open irrigated-tip $\mathrm{RF}$ ablation

\begin{tabular}{|c|c|c|c|c|}
\hline & \multicolumn{2}{|c|}{ Lowest Cost } & \multicolumn{2}{|c|}{ Highest Cost } \\
\hline & Manufacturer & Cost: lowest & Manufacturer & Cost: highest \\
\hline $\begin{array}{l}\text { Open irrigated tip } \\
\text { ablation catheter } \\
+ \text { cable+ } \\
\text { irrigation tubing }\end{array}$ & $\begin{array}{l}\text { St. Jude Cool } \\
\text { Path } \\
\text { unidirectional* }\end{array}$ & $\$ 1795^{\star}$ & $\begin{array}{c}\text { Biosense Webster } \\
\text { ThermoCool } \\
\text { bidirectional } \\
\text { navigation }\end{array}$ & $\$ 3490$ \\
\hline $\begin{array}{l}\text { Required } \\
\text { navigation patch }\end{array}$ & St. Jude NavX ${ }^{T M}$ & $\$ 1045$ & $\begin{array}{c}\text { Biosense-Webster } \\
\text { Carto }^{\mathrm{TM}}\end{array}$ & $\$ 420$ \\
\hline $\begin{array}{l}\text { Duodecapolar } \\
\text { catheter+cable }\end{array}$ & $\begin{array}{c}\text { St. Jude } \\
\text { Livewire } \\
\text { unidirectional }\end{array}$ & $\$ 1172 \nmid$ & $\begin{array}{c}\text { Boston Scientific } \\
\text { Blazer } \otimes X 20 \\
\text { bidirectional }\end{array}$ & $\$ 1425 t$ \\
\hline $\begin{array}{l}\text { Intracardiac } \\
\text { ultrasound }\end{array}$ & $\begin{array}{c}\text { Boston Scientific } \\
\text { Ultra ICE }{ }^{\mathrm{TM}} \\
\text { rotational+ dock }\end{array}$ & $\$ 1050 \S$ & $\begin{array}{c}\text { Biosense Webster } \\
\text { AcuNav }{ }^{\mathrm{TM}} \text { phased } \\
\text { array }\end{array}$ & $\$ 2800$ \\
\hline $\begin{array}{l}\text { Transseptal } \\
\text { needle }\end{array}$ & Biosense Webster & $\$ 175$ & $\begin{array}{c}\text { Baylis NRG }^{\mathrm{TM}}+ \\
\text { cable }\end{array}$ & $\$ 645$ \\
\hline $\begin{array}{l}\text { Transseptal } \\
\text { sheath }\end{array}$ & $\begin{array}{l}\text { Biosense Webster } \\
\text { single transseptal }\end{array}$ & $\$ 175$ & $\begin{array}{l}\text { St. Jude Agilis }{ }^{\mathrm{TM}} \\
\text { double transseptal }\end{array}$ & $\$ 1998$ \\
\hline $\begin{array}{l}\text { Circular catheter } \\
+ \text { cable }\end{array}$ & $\begin{array}{l}\text { Biosense Webster } \\
\text { Lasso } 110 \text { pole } \\
\text { fixed diameter }\end{array}$ & $\$ 1225 t$ & $\begin{array}{c}\text { Biosense Webster } \\
\begin{array}{c}\text { Lasso® variable } \\
\text { diameter }\end{array}\end{array}$ & $\$ 1825\}$ \\
\hline Total cost & & $\$ 6,637$ & & $\$ 12,603$ \\
\hline $\begin{array}{l}\text { Percent } \\
\text { above lowest } \\
\text { cost }\end{array}$ & & & & $89.9 \%$ \\
\hline
\end{tabular}


ablation catheter advancement system and \$250/case circular mapping catheter drive. Figure 3 shows the lowest and highest cost projections for an RF ablation using MNS. The system requires special catheters which cost $\$ 3,590$ including cables and irrigation tubing and can only utilize the Carto $^{\text {TM }} 3$ mapping system. The lowest- and highest-cost scenarios for a MNS ablation are \$12,261 and \$15,464. These costs for MNS ablation are 84.7 and $133 \%$ higher than the lowest-cost RF equipment.

\subsection{Cost of cryoballoon ablation without touch-up}

Figure 4(a) shows the cost of the Medtronic Arctic Front ${ }^{\circledR}$ cryoablation balloon system assuming PVs are completely isolated by the balloon requiring no additional touch-up or mapping system. We assumed a 5-year lifespan for the CryoConsole and 200 cases/year. The lowest-cost scenario includes the use of a single cryoballoon, a steerable FlexCath ${ }^{\circledR}$ sheath with the Achieve ${ }^{\mathrm{TM}}$ circular catheter, a St. Jude Livewire ${ }^{\mathrm{TM}}$ unidirectional duodecapolar CS catheter, a rotational ultrasound, and a standard transseptal needle for a total cost of $\$ 12,847$. The highest-cost cryoballoon scenario without touch-up includes the addition of the Acu$\mathrm{Nav}^{\mathrm{TM}}$ phased array ICE and the Baylis NRG ${ }^{\mathrm{TM}}$ RF needle for transseptal puncture for a total cost of $\$ 15,320$. These lowest and highest projected costs range from $93.6 \%$ to $131 \%$ above the lowest cost for RF ablation.

3.7 Cryoballoon ablation with focal cryoablation touch-up and/or RF touch-up

The costs of a cryoballoon ablation with focal cryoablation touch-up for the lowest- or highest-cost scenarios is similar to that for the balloon only ablation but requires the addition of a Freezor ${ }^{\circledR}$ MAX focal cryoablation catheter for $\$ 3,095$. The lowest cost estimate requires repeated removal of the cryoballoon and insertion of the focal ablation catheter through the same sheath. The highest-cost estimate assumes the addition of a variable-diameter circular mapping catheter used independently of the cryoballoon through a steerable sheath. The total lowest and highest costs of $\$ 15,942$ and $\$ 22,284$ were $140 \%$ and $236 \%$ higher than the lowest-cost RF ablation.

Figure 4(b) shows the costs of the lowest and highest cryoballoon ablation assuming RF is needed for additional focal-segmental isolation of PVs or to make any additional lines/lesions. These costs are the same as for isolated cryoballoon ablation but require the added cost of open irrigated-tip RF catheters and cables. The lowest-cost scenario assumes that one repeatedly exchanges the cryoballoon and Achieve ${ }^{\mathrm{TM}}$
Fig. 3 Lowest and highest estimated equipment costs for performing RFAF ablation using Stereotaxis NIROBE ${ }^{\circledR}$ Remote Magnetic Navigation System

\begin{tabular}{|c|c|c|c|c|}
\hline & \multicolumn{2}{|c|}{ Lowest Cost } & \multicolumn{2}{|c|}{ Highest Cost } \\
\hline & Manufacturer & Cost: lowest & Manufacturer & Cost: highest \\
\hline $\begin{array}{l}\text { Open irrigated tip } \\
\text { ablation catheter }+\mathrm{B} \\
\text { cable+ irrigation } \\
\text { tubing }\end{array}$ & $\begin{array}{l}\text { Biosense Webster } \\
\text { RMT }\end{array}$ & $\$ 3590$ & $\begin{array}{c}\text { Biosense Webster } \\
\text { ThermoCool } \\
\text { bidirectional } \\
\text { navigation }\end{array}$ & $\$ 3590$ \\
\hline Navigation patch & $\begin{array}{c}\text { Biosense Webster } \\
\text { Carto }^{\mathrm{TM}}\end{array}$ & $\$ 420$ & $\begin{array}{c}\text { Biosense Webster } \\
\text { Carto IM }^{\text {IM }}\end{array}$ & $\$ 420$ \\
\hline $\begin{array}{l}\text { Duodecapolar } \\
\text { catheter+cable }\end{array}$ & $\begin{array}{c}\text { St. Jude } \\
\text { Livewire }^{\mathrm{TM}} \\
\text { unidirectional }\end{array}$ & $\$ 1172^{*}$ & $\begin{array}{c}\text { Boston Scientific } \\
\text { Blazer } \otimes X 20 \\
\text { bidirectional }\end{array}$ & $\$ 1425^{*}$ \\
\hline $\begin{array}{l}\text { Intracardiac } \\
\text { ultrasound }\end{array}$ & $\begin{array}{c}\text { Boston Scientific } \\
\text { Ultra ICE }{ }^{\mathrm{TM}} \\
\text { rotational+ dock }\end{array}$ & $\$ 1050 \nmid$ & $\begin{array}{c}\text { Biosense Webster } \\
\text { AcuNav }{ }^{\mathrm{TM}} \text { phased } \\
\text { array }\end{array}$ & $\$ 2800$ \\
\hline $\begin{array}{l}\text { Transseptal } \\
\text { needle }\end{array}$ & BiosenseWebster & $\$ 175$ & $\begin{array}{c}\text { Baylis NRG }{ }^{T M}+ \\
\text { cable }\end{array}$ & $\$ 645$ \\
\hline $\begin{array}{l}\text { Transseptal } \\
\text { sheathx2 }\end{array}$ & Biosense Webster & $\$ 350$ & St. Jude $\times 2$ & $\$ 480$ \\
\hline $\begin{array}{l}\text { Circular catheter }+ \\
\text { cable }\end{array}$ & $\begin{array}{l}\text { Biosense Webster } \\
\text { Lasso }(10 \text { pole } \\
\text { fixed diameter }\end{array}$ & $\$ 1225^{\star}$ & $\begin{array}{l}\text { Biosense Webster } \\
\text { Lasso } \otimes 20 \text { pole } \\
\text { variable diameter }\end{array}$ & $\$ 1825^{\star}$ \\
\hline $\begin{array}{l}\text { NIROBE® } \\
\text { Magnets } \\
\text { Cost/case }\end{array}$ & Stereotaxis & $\$ 2829$ & Stereotaxis & $\$ 2829$ \\
\hline $\begin{array}{l}\text { Catheter } \\
\text { advancement } \\
\text { system }\end{array}$ & Stereotaxis & $\$ 1200$ & Stereotaxis & $\$ 1200$ \\
\hline $\begin{array}{l}\text { V Drive Catheter } \\
\text { advancement } \\
\text { system }\end{array}$ & Stereotaxis & $\$ 250$ & Stereotaxis & $\$ 250$ \\
\hline Total cost & & $\$ 12,261$ & & $\$ 15,464$ \\
\hline $\begin{array}{l}\text { Percent above } \\
\text { lowest cost }\end{array}$ & & $84.7 \%$ & & $133 \%$ \\
\hline
\end{tabular}


Fig. 4 (a) Lowest and highest estimated equipment costs for performing AF balloon cryoablation without touch-up or 3D mapping system. (b) Lowest and highest estimated equipment costs for performing AF balloon cryoablation with $\mathrm{RF}$ touch-up requiring $3 \mathrm{D}$ mapping system (a)

\begin{tabular}{|c|c|c|c|c|}
\hline & \multicolumn{2}{|c|}{ Lowest Cost } & \multicolumn{2}{|c|}{ Highest Cost } \\
\hline & Manufacturer & Cost: lowest & Manufacturer & Cost: highest \\
\hline Cryoballoon & $\begin{array}{l}\text { Medtronic Arctic } \\
\text { Front } 8\end{array}$ & $\$ 6500$ & $\begin{array}{l}\text { Medtronic Arctic } \\
\text { Front } 8\end{array}$ & $\$ 6500$ \\
\hline Cryocable & Medtronic & $\$ 215$ & Medtronic & $\$ 215$ \\
\hline $\begin{array}{l}\text { Cryocoaxial } \\
\text { umbilical }\end{array}$ & Medtronic & $\$ 145$ & Medtronic & $\$ 145$ \\
\hline $\begin{array}{l}\text { Cryoablation } \\
\text { system cost/case }\end{array}$ & $\begin{array}{c}\text { Medtronic } \\
\text { CryoConsole }^{*}\end{array}$ & $\$ 265$ & $\begin{array}{c}\text { Medtronic } \\
\text { CryoConsole }^{*}\end{array}$ & $\$ 265$ \\
\hline Balloon sheath & $\begin{array}{l}\text { Medtronic } \\
\text { Flexcath® }\end{array}$ & $\$ 1800$ & $\begin{array}{l}\text { Medtronic } \\
\text { Flexcath }{ }^{\circledR}\end{array}$ & $\$ 1800$ \\
\hline $\begin{array}{l}\text { Circularmapping } \\
\text { catheter }\end{array}$ & $\begin{array}{l}\text { Medtronic } \\
\text { Achieve }^{\mathrm{TM}}\end{array}$ & $\$ 1525 t$ & $\begin{array}{l}\text { Medtronic } \\
\text { Achieve }^{\mathrm{TM}}\end{array}$ & $\$ 1525 t$ \\
\hline $\begin{array}{l}\text { Duodecapolar } \\
\text { catheter+cable }\end{array}$ & $\begin{array}{c}\text { St. Jude } \\
\text { Livewire }^{\mathrm{TM}} \\
\text { unidirectional }\end{array}$ & $\$ 1172 \nmid$ & $\begin{array}{c}\text { Boston Scientific } \\
\text { Blazer } ® X 20 \\
\text { bidirectional }\end{array}$ & $\$ 1425 t$ \\
\hline $\begin{array}{l}\text { Intracardiac } \\
\text { ultrasound }\end{array}$ & $\begin{array}{c}\text { Boston Scientific } \\
\text { Ultra ICE } \\
\text { rotational+ dock }\end{array}$ & $\$ 1050 \S$ & $\begin{array}{c}\text { Biosense Webster } \\
\text { AcuNav }{ }^{\mathrm{TM}} \\
\text { phased-array }\end{array}$ & $\$ 2800$ \\
\hline $\begin{array}{l}\text { Transseptal } \\
\text { needle }\end{array}$ & Biosense Webster & $\$ 175$ & $\begin{array}{l}\text { Baylis NRG }{ }^{\mathrm{IM}}+ \\
\text { cable }\end{array}$ & $\$ 645$ \\
\hline Total & & $\$ 12,847$ & & $\$ 15,320$ \\
\hline $\begin{array}{l}\text { Percent above } \\
\text { lowest cost }\end{array}$ & & $93.6 \%$ & & $131 \%$ \\
\hline
\end{tabular}

(b)

\begin{tabular}{|c|c|c|c|c|}
\hline & \multicolumn{2}{|c|}{ Lowest Cost } & \multicolumn{2}{|c|}{ Highest Cost } \\
\hline & Manufacturer & Cost: lowest & Manufacturer & Cost:highest \\
\hline Cryoballoon & $\begin{array}{c}\text { Medtronic Arctic } \\
\text { Front }(8\end{array}$ & $\$ 6500$ & $\begin{array}{c}\text { Medtronic Arctic } \\
\text { Front }\end{array}$ & $\$ 6500$ \\
\hline Cryocable & Medtronic & $\$ 215$ & Medtronic & $\$ 215$ \\
\hline $\begin{array}{l}\text { Cryocoaxial } \\
\text { umbilical }\end{array}$ & Medtronic & $\$ 145$ & Medtronic & $\$ 145$ \\
\hline $\begin{array}{l}\text { Cryoablation } \\
\text { system cost/case }\end{array}$ & $\begin{array}{l}\text { Medtronic } \\
\text { CryoConsole }\end{array}$ & $\$ 265$ & $\begin{array}{l}\text { Medtronic } \\
\text { CryoConsole }\end{array}$ & $\$ 265$ \\
\hline Balloon sheath & $\begin{array}{l}\text { Medtronic } \\
\text { Flexcath® }\end{array}$ & $\$ 1800$ & $\begin{array}{l}\text { Medtronic } \\
\text { Flexcath } §\end{array}$ & $\$ 1800$ \\
\hline $\begin{array}{l}\text { Circularmapping } \\
\text { catheter + cable }\end{array}$ & $\begin{array}{l}\text { Medtronic } \\
\text { Achieve }^{\mathrm{TM}}\end{array}$ & $\$ 1525^{*}$ & $\begin{array}{l}\text { Medtronic } \\
\text { Achieve }^{\mathrm{TM}}\end{array}$ & $\$ 1525^{*}$ \\
\hline $\begin{array}{l}\text { Duodecapolar } \\
\text { catheter + cable }\end{array}$ & $\begin{array}{l}\text { St. Jude } \\
\text { Livewire } \\
\text { unidirectional }\end{array}$ & $\$ 1172^{*}$ & $\begin{array}{c}\text { Boston Scientific } \\
\text { Blazer DX20 } \\
\text { bidirectional }\end{array}$ & $\$ 1425^{*}$ \\
\hline $\begin{array}{l}\text { Intracardiac } \\
\text { ultrasound }\end{array}$ & $\begin{array}{c}\text { Boston Scientific } \\
\text { Ultra ICE TM } \\
\text { rotational+ dock }\end{array}$ & $\$ 1050 t$ & $\begin{array}{c}\text { Biosense Webster } \\
\text { AcuNav } \\
\text { phased array }\end{array}$ & $\$ 2800$ \\
\hline $\begin{array}{l}\text { Transseptal } \\
\text { needle }\end{array}$ & Biosense Webster & $\$ 175$ & $\begin{array}{c}\text { Baylis NRG }{ }^{\mathrm{IM}}+ \\
\text { cable }\end{array}$ & $\$ 645$ \\
\hline $\begin{array}{l}\text { Open irrigated tip } \\
\text { ablation catheter }+ \\
\text { cable + tubing }\end{array}$ & $\begin{array}{c}\text { St. Jude Cool } \\
\text { Path } \\
\text { unidirectional }\end{array}$ & $\$ 1785 \S$ & $\begin{array}{c}\text { Biosense Webster } \\
\text { ThermoCool } 8 \\
\text { bidirectional } \\
\text { navigation }\end{array}$ & $\$ 3490$ \\
\hline $\begin{array}{l}\text { Required } \\
\text { navigation patch }\end{array}$ & St. Jude NavX ${ }^{T M}$ & $\$ 1045$ & $\begin{array}{c}\text { Biosense Webster } \\
\text { Carto }^{\mathrm{TM}}\end{array}$ & $\$ 420$ \\
\hline $\begin{array}{l}\text { Transseptal } \\
\text { sheath (second } \\
\text { one) }\end{array}$ & & & St. Jude Agilis ${ }^{\mathrm{TM}}$ & $\$ 999$ \\
\hline $\begin{array}{l}\text { Circular catheter }+ \\
\text { cable }\end{array}$ & & & $\begin{array}{l}\text { Biosense Webster } \\
\text { Lasso } \otimes 20 \text { pole } \\
\text { variable diameter }\end{array}$ & $\$ 1825^{\star}$ \\
\hline Total & & $\$ 15,677$ & & $\$ 22,054$ \\
\hline $\begin{array}{l}\text { Percent above } \\
\text { lowest cost }\end{array}$ & & $136 \%$ & & $232.0 \%$ \\
\hline $\begin{array}{l}\text { Assumes } 10 \text { cable } \\
\text { to Begin Saving = }\end{array}$ & $\begin{array}{l}\text { euses; }+ \text { Numbe } \\
1 \text { cases }\end{array}$ & led to $B e c$ & ving $=82.6 \mathrm{cas}$ & Number $\mathrm{Ne}$ \\
\hline
\end{tabular}


mapping catheter with the RF ablation catheter through the cryoballoon sheath. The highest-cost scenario includes the use of a mapping system, 20-pole variable-diameter circular catheter, and additional steerable sheath placed in the left atrium. The lowest and highest estimated costs of cryoballoon ablation with RF touch up range from $\$ 15,667-\$ 22,054$, amounting to $136 \%$ to $232 \%$ above the lowest cost for RF ablation.

\section{Discussion}

These simulated cost scenarios demonstrate that, depending upon the ablation equipment combination used, the cost of the special equipment to perform an $\mathrm{AF}$ ablation ranges from $\$ 6,637-\$ 22,054$. The difference in cost of the special equipment required for $\mathrm{AF}$ ablation is largely driven by physician choice. The average age of our patients at Sequoia Hospital undergoing AF ablation is now $64.3 \pm 10$ years [11], indicating that approximately half of our patients will have Medicare as their primary insurance. For Medicare patients, whose average reimbursment is $\$ 10,338$ [15], the financial long-term sustainability of an AF ablation program would be challenging using anything but a variation of our lowest-priced RF ablation scenarios. Since we have only described a small part of the actual AF ablation costs, the addition of personnel costs, cath lab costs, floor monitoring, post-ablation care, and facility overhead would make it difficult to break-even at our lowest-priced equipment costs. This suggests that current Medicare reimbursement for AF ablation may be far below the cost of performing this procedure and may need upward adjustment in the future. DRG 251 is for "percutaneous cardiovascular procedure without coronary artery stent," an old angioplasty code, and does not reflect the complexity and costs associated with AF ablation. There is little scientific evidence suggesting that the equipment utilized in our more expensive ablation scenarios is safer or more efficacious than less expensive scenarios. Although phased-array ICE, bidirectional RF ablation catheters, steerable transseptal sheaths, MNS, and cryoballoons are often perceived as important for performing successful RF AF ablation, these choices are largely the physician's personal preferences. Physicians performing AF ablations may have different skill sets and need to be given some latitude regarding which tools work best in their hands. For example, at Sequoia Hospital, we generally follow an equipment scenario close to the one for the lowest-cost RF ablation costs. However, we routinely use the Baylis NRG ${ }^{\mathrm{TM}} \mathrm{RF}$ transseptal needle which adds $\$ 470$ to the costs, since we have shown this needle to be both safer in terms of fewer tamponades and more efficacious with regard to more successful and faster septal crossing than using the standard transseptal needle [16]. We generally prefer a bidirectional RF ablation catheter and a variable rather than fixed-diameter circular catheter to verify $\mathrm{PV}$ isolation. Our total list price equipment cost is $\$ 7,968$, which is $20 \%$ above the lowest-cost scenario for RF ablation. In Europe, many physicians do not use ICE for transseptal puncture due to cost considerations. There is little evidence that robotic MNS perform better or more safely than manual catheter manipulation. In one study which randomized patients to manual catheter manipulation or to MNS ablation, patient radiation exposure and procedural times were higher with MNS, and there was no difference in 1-year outcomes [12]. Although cryoballoon technology is considerably more expensive than RF technology, there is little evidence to suggest increased efficacy or safety for cryoablation. A recent literature review indicated the singleprocedure cryoablation $\mathrm{AF}$-free rates at 1 year ranged from 69.9 to $76.7 \%$ for paroxysmal AF and 41.9 to $48.4 \%$ for persistent AF [17]. These numbers are similar to outcomes from a number of centers using RF energy [6-11].

Given the large variation in cost of equipment for performing $\mathrm{AF}$ ablations, more expensive technologies will need to prove superiority to existing less expensive technologies with regard to long-term efficacy and/or patient safety. In order to make the most cost-effective equipment choices, physicians need considerably more studies randomizing patients to various ablation equipment types to better determine comparative safety and efficacy. In the coming era of severe healthcare cost constraints, being "noninferior" will not be sufficient if costs are significantly higher. Evaluation and adoption of new technologies should consider costeffectiveness in addition to efficacy and safety. As physicians partner with hospitals, either through employment or single payment reimbursement schemes, the cost of equipment utilized will become increasingly as important to the physicians as it may be to their hospitals.

\subsection{Limitations}

We assumed a large amount of equipment was already in place and did not include any personnel or hospital costs for continued patient care following the ablation procedure. These costs would considerably increase the total cost of an ablation procedure beyond those we have considered. For our analysis, we used list prices, and it could, at times, be surprisingly difficult to obtain these numbers. The manufacturers who provided our list price data indicated that they routinely give approximately a $20 \%$ contractual discount to high-volume users. We could not include these discounts in our analysis as they can vary widely from center to center, and all of the hospital-manufacturer pricing contracts are confidential. We also assumed 200 cases per year for our magnetic robotic navigation system. If this caseload is not achieved, the costs of this technology would be considerably higher than our scenarios suggest. We did not do a 
formal cost/benefit analysis as there are little data in the literature from which to derive comparative benefit data, and any analysis would require so many assumptions that the conclusions would be of limited value. We also did not consider reuse of diagnostic catheters and the phased-array ultrasound catheter. Catheter reuse can also lower the equipment costs and should be considered by physicians when feasible to reduce equipment costs. It was hard to factor reuse into our analysis, as informal polls of $\mathrm{AF}$ ablation centers found a great variation in catheters reused and the number of times reused. Since it is not feasible to reuse irrigated-tip ablation catheters or cryoballoons, reuse of any item other than the phased-array ultrasound catheter should have only a small impact on our overall equipment cost analysis. Finally, we realize many physicians might use slightly different catheters combinations (i.e., decapolar or quadripolar CS) than we chose for our scenarios and that these choices might alter the equipment cost analysis.

\section{Conclusions}

Physicians have many choices of AF ablation equipment. These equipment costs for the scenarios we evaluated ranged from $\$ 6,637$ to $\$ 22,284$ per case. The specifics of each catheter combination we chose are not as important as the concept that ablationists should begin to critically look at their own equipment costs and try to determine if they believe the benefits to using them outweigh the additional costs. More expensive technology should markedly increase procedural efficacy and/or patient safety to justify the increase in procedural equipment costs.

Acknowledgments Glenda Rhodes assisted with manuscript management and Patricia Barberini, R.N., provided critical manuscript review.

Disclosures Dr. Mead: Advisory boards: Medtronic, iRhythm, Proteus Biomedical, Voyage Medical. Stock options: iRhythm, Voyage Medical and Proteus Biomedical. Director: iRhythm and Voyage Medical. Dr. Patrawala: Consultant/stock options: Voyage Medical. Speaker: St. Jude Medical. Dr. Engel: Speaker: Medtronic. Dr. Kong: Advisory Boards: Medtronic, Biotronik. Research grant: Biotronik. Drs. Winkle, Mead, Engel and Patrawala: Investigators: Medtronic and Sanofi-Aventis. Dr. Winkle no other disclosures.

Open Access This article is distributed under the terms of the Creative Commons Attribution License which permits any use, distribution, and reproduction in any medium, provided the original author(s) and the source are credited.

\section{References}

1. Haissaguerre, M., Jais, P., Shah, D., Takahashi, A., Hocini, M., Quiniou, G., et al. (1998). Spontaneous initiation of atrial fibrillation by ectopic beats originating from the pulmonary veins. The New England Journal of Medicine, 339, 659-666.
2. Calkins, H., Kuck, K. H., Cappato, R., Brugada, J., Camm, A. J., Chen, S., et al. (2012). 1012 HRS/EHRA/ECAS expert consensus statement on catheter and surgical ablation of atrial fibrillation: recommendations for patient selection, procedural techniques, patient management and follow-up, definitions, endpoints, and research trial design. Journal of Interventional Cardiac Electrophysiology, 33, 171-257.

3. Katritsis, D. G., Giazitzoglou, E., Zografos, T., Pokushalov, E., Po, S. S., \& Camm, A. J. (2011). Rapid pulmonary vein isolation combined with autonomic ganglia modification: A randomized study. Heart Rhythm, 8, 672-678.

4. Winkle, R. A., Mead, R. H., Engel, G., \& Patrawala, R. A. (2011). Atrial fibrillation ablation: Perpetual motion of open irrigated tip catheters at 50 watts is safe and improves outcomes. Pacing And Clinical Elecrophysiology, 34, 531-539.

5. Winkle, R. A., Mead, R. H., Engel, G., \& Patrawala, R. A. (2011). Safety of lower activated clotting times during atrial fibrillation ablation using open irrigated tip catheters and a single transseptal puncture. The American Journal of Cardiology, 107, 704-708.

6. Tzou, W. S., Marchlinski, F. E., Zado, E. S., Lin, D., Dixit, S., Callans, D. J., et al. (2010). Long-term outcome after successful catheter ablation of atrial fibrillation. Circulation Arrhythmia and Electrophysiology, 3, 237-242.

7. Wokhlu, A., Hodge, D. O., Monahan, K. H., Asirvatham, S. J., Friedman, P. A., Munger, T. M., et al. (2010). Long-term outcome of atrial fibrillation ablation: Impact and predictors of very late recurrence. Journal of Cardiovascular Electrophysiology, 21, 1071-1076.

8. Winkle, R. A., Mead, R. H., Engel, G., \& Patrawala, R. A. (2011). Long term results of atrial fibrillation ablation: The importance of all initial ablation failures undergoing a repeat ablation. American Heart Journal, 162, 193-2010.

9. Winkle, R. A., Mead, R. H., Engel, G., Kong, M. H., \& Patrawala, R. A. (2012). Prior antiarrhythmic drug use and the outcome of atrial fibrillation ablation. Europace, 14, 646-652.

10. Patel, D., Mohanty, P., Di Biase, L., Sanchez, J. E., Shaheen, M. H., Burkhardt, J. D., et al. (2010). Outcomes and complications of catheter ablation for atrial fibrillation in females. Heart Rhythm, 7, $167-172$.

11. Winkle, R. A., Mead, R. H., Engel, G., Kong, M. H., \& Patrawala, R. A. (2012). Trends in atrial fibrillation ablation: Have we maximized the current paradigms? Journal of Interventional Cardiac Electrophysiology, 34, 115-123.

12. Hoyt, H., Bhonsale, A., Chilukuri, K., Alhumaid, F., Needleman, M., Edward, D., et al. (2011). Complications arising from catheter ablation of atrial fibrillation: Temporal trends and predictors. Heart Rhythm, 8, 1869-1874.

13. Miyazaki, S., Shah, A. J., Xhaët, O., Derval, N., Matsuo, S., Wright, M., et al. (2010). Remote magnetic navigation with irrigated tip catheter for ablation of paroxysmal atrial fibrillation. Circulation Arrhythmia and Electrophysiology, 3, 585-589.

14. Hlivák, P., Mlčochová, H., Peichl, P., Čihká, R., Wichterle, D., \& Kautzner, J. (2011). Robotic navigation in catheter ablation for paroxysmal atrial fibrillation: Midterm efficacy and predictors of postablation arrhythmia recurrences. Journal of Cardiovascular Electrophysiology, 22, 534-540.

15. Biosense Webster Reimbursement and Coding Guide. http://www. biosensewebster.com/professionalResources/reimbursement.aspx, Accessed May 20, 2012.

16. Winkle, R. A., Mead, R. H., Engel, G., \& Patrawala, R. A. (2011). The use of a radiofrequency needle improves the safety and efficacy of transseptal puncture for atrial fibrillation ablation. Heart Rhythm, 8, 1411-1415.

17. Andrade, J. G., Khairy, P., Guerra, P. G., Deyell, M. W., Rivare, L., Macle, L., et al. (2011). Efficacy and safety of cryoballoon ablation for atrial fibrillation: A systematic review of published studies. Heart Rhythm, 8, 1444-1451. 